\title{
European Innovation Partnership on Water: opportunità per l'innovazione nel settore della risorsa idrica
}

\author{
Rudy Rossetto \\ Istituto di Scienze della Vita, Scuola Superiore Sant’Anna \\ r.rossetto@sssup.it
}

In un momento in cui la crisi economica ha oramai presa in tutti i settori, non ultimo quello relativo agli utilizzi e gestione della risorsa idrica, ogni iniziativa che tenda a sostenere il trasferimento dell'innovazione nel privato e nel pubblico non può che essere ben vista. Ciò è particolarmente vero in Italia per i settori dell'economia reale che operano nel campo dell'acqua, che, complice spesso una formazione universitaria non proprio al passo con i tempi, mancano degli adeguati strumenti per trasferire i risultati della ricerca in innovazione e soffrono sul mercato le più dinamiche e attrezzate realtà europee. Lo stesso vale anche per il settore pubblico, dove è necessaria e fondamentale una quantomeno "attualizzazione" in primis del capitale umano.

Le partnership europee per l'innovazione (European Innovation Partnerships - EIPs; 2013) hanno l'obiettivo di accelerare l'innovazione sì da contribuire a risolvere le grandi sfide sociali, migliorare la competitività dell'Europa e supportare la creazione di posti di lavoro. Le EIPs tentano di raggiungere questo obiettivo riunendo competenze e risorse, portando insieme attori pubblici e privati, a livello comunitario, nazionale e regionale, attraverso l'individuazione di misure che tendano a facilitare l'incontro tra domanda ed offerta di innovazione.

La European Innovation Partnership on Water (EIPW; 2013) è una iniziativa promossa dalla Commissione Europea all'interno del programma Innovation Union (2013). Quest'ultima altro non è che la strategia dell'Unione per la creazione di un ambiente per l'innovazione che possa aiutare idee eccellenti a trasformarsi in prodotti e servizi sempre per la crescita economica e la creazione di posti di lavoro.

La EIPW ha l'obiettivo di facilitare lo sviluppo di soluzioni innovative per risolvere le principali sfide relative al tema acqua a livello europeo e globale. Allo stesso tempo, intende supportare la creazione di opportunità per il mercato per queste innovazioni, sia all'interno che all'esterno della EU, rimuovendo le barriere che si frappongono tra esse e l'ingresso nel mercato e nel settore pubblico. Il programma ha avuto inizio nel Maggio 2013 con il principale obiettivo di iniziare a promuovere processi di collaborazione per il cambiamento e l'innovazione sia nel pubblico che nel privato, nelle organizzazioni non-governative e più in generale nella società civile. Tutto ciò è messo in pratica attraverso l'istituzione di Azioni (Actions) e Gruppi di Lavoro (Working Groups).

Per raggiungere gli obiettivi sopra esposti è necessario acquisire inizialmente un'ampia visione sulle necessità di innovazione. Questi bisogni includeranno nuovi prodotti, ma anche processi e metodi di lavoro da adottare sia nel settore pubblico sia nel privato. Per rimuovere le barriere all'innovazione e per assicurare l'applicazione di soluzioni innovative, è necessario integrare la ricerca e le soluzioni tecnologiche (in primis soluzioni ICT) con motori quali la finanza, la assunzione di consapevolezza da parte dell'opinione pubblica, strumenti di governance e la formazione del capitale umano. E' inoltre necessaria una visione globale, poiché numerose attività di innovazione avvengono in partenariati o hanno obiettivi di carattere internazionale. Per il 2020, EIPW vuole raggiungere l'obiettivo principale di identificare, promuovere e stimolare il mercato, testare e applicare su vasta scala soluzioni per circa nove aree prioritarie, principali sfide per il tema acqua, che presentano frequenti connessioni tra di loro.

Queste aree prioritarie sono:

1) Riuso e riciclo dell'acqua: parte rilevante dell'approccio integrato alla gestione della risorsa idrica per ridurre costi, recuperare risorse e dimostrare sostenibilità ambientale. L'uso di risorse non convenzionali può supportare la fornitura di acqua di buona qualità per specifici processi diminuendo la domanda di energia, i costi di trattamento e l'impatto sull'ambiente. Le barriere al riuso e riciclo, oltre ad essere di tipo tecnologico (mancanza di un mercato strutturato per il recupero di nutrienti, processi industriali non adeguati all'utilizzo di acque con caratteristiche di qualità variabili), sono legate alla limitata capacità delle istituzioni di sostenere misure per il riciclo ed il riuso, mancanza di incentivi finanziari ed una sfavorevole percezione da parte della opinione pubblica, anche in assenza di standard condivisi e armonizzati all'interno della EU.

2) Sistemi di trattamento delle acque: priorità complementare alla precedente, vuole porre in evidenza la necessità di disporre di sistemi di trattamento efficienti che permettano di passare dal trattamento per lo scarico nei corpi idrici alla fornitura di acqua per gli usi più diversi.

3) Nesso tra acqua e energia: è un nesso ben conosciuto, ma poco affrontato dalle politiche europee sull'acqua e l'energia. I sistemi di trattamento delle acque sono comunemente energivori e mancano di tecnologie di processo a basso consumo, mentre in molti casi l'utilizzo delle rinnovabili può nascondere utilizzi poco efficienti della risorsa idrica. Tecnologie a basso consumo sono inoltre oramai necessarie anche nel campo dell'irrigazione nell'agricoltura moderna.

4) Gestione delle alluvioni e della siccità: questi due momenti, espressione estrema oramai sempre più frequente del ciclo 
dell'acqua, hanno enormi conseguenze ambientali, sociali ed economiche e i cambiamenti climatici in atto ne peggioreranno gli impatti. La gestione degli eventi estremi richiede un approccio integrato del rischio che includa la prevenzione, la preparazione, la risposta e la successiva ripresa. Educazione, assunzione di consapevolezza e capacità di comunicazione diretta all'opinione pubblica ed agli attori economici sono indispensabili per affrontare il cambiamento. È quindi necessaria l'integrazione dello stato dell'arte dei sistemi informativi per la gestione del rischio e delle crisi, che includano oltre agli aspetti tecnologici la partecipazione dei principali attori, e la loro reale applicazione.

5) Servizi ecosistemici: è necessario un approccio innovativo che vada a dare un valore ai benefici che i sistemi socio-economici ricevono dagli ecosistemi al fine di includerli nella gestione integrata della risorsa idrica. La scarsa conoscenza sul ruolo degli ecosistemi nella fornitura di servizi collegati al tema acqua ha bisogno di essere colmata e trasformata in un concetto applicabile e replicabile su larga scala. La barriera principale da superare è insita nella frammentazione di responsabilità e conoscenza dispersa in varie discipline scientifiche, autorità amministrative ed agenzie ambientali.

6) Governo dell'acqua: l'applicazione di tecnologie innovative è praticamente impossibile senza considerare gli aspetti relativi alla governance. Innovare il governo dell'acqua è necessario per meglio realizzare obiettivi di pubblico interesse, mentre la gestione delle innovazioni è cruciale per aiutarne l'applicazione. Il superamento della attuale frammentazione delle realtà implicate nella gestione della risorsa idrica (in Italia localmente si può arrivare fino a 7/8 istituzioni) è necessario per aumentare l'interesse pubblico ed attrarre investimenti privati.

7) Sistemi di supporto alle decisioni e monitoraggio: la gestione della risorsa idrica in Europa sta passando dall'esser focalizzata sull'approvvigionamento alla gestione della domanda. Ciò porta a considerare nella gestione non solo la parte idrologica, ma un approccio olistico che includa gli aspetti ecologici e socio-economici. Modelli e sistemi di supporto alle decisioni per la gestione della risorsa (Modelling and Decision Support Systems - MDSS) unitamente al monitoraggio sono strumentali a supportare questo cambiamento.

8) Finanziare l'innovazione: l'accesso limitato ad appropriate forme di finanziamento può restringere l'innovazione nel settore della risorsa idrica, sia all'interno che all'esterno della EU. Una sfida chiave consiste quindi nell'incrementare il flusso finanziario. Fattori cruciali sono costituiti da una appropriata percezione del rischio verso la remunerazione, dove una miscela di contributi, prestiti e altri strumenti finanziari può giocare un ruolo importante. Allo stesso tempo strumenti non finanziari quali incentivi, formazione e diffusione della conoscenza possono aiutare a sbloccare i flussi finanziari verso il settore. Tra gli obiettivi di questa area prioritaria vi è quello di supportare le piccole medie imprese ad acquisire le risorse per rispondere alle opportunità offerte dal mercato e supportarle nell'accesso ai fondi già disponibili.
9) Smart technologies: tutte quelle tecnologie che, altamente efficienti e interdisciplinari, permettono di compiere un balzo in avanti nella gestione della risorsa. Sensori e metodi di gestione delle reti sono esempi di tali tecnologie che impattano tutte le precedenti aree prioritarie.

Al cuore della EIPW sono i Gruppi di Azione (Action groups). Multi-stakeholder e su base volontaria sono la base dell'iniziativa e ne costituiscono l'elemento centrale della fase di implementazione. I primi nove Gruppi di Azione sono stati selezionati dalla EU nel Maggio 2013; una seconda lista è stata recentemente approvata nel mese di Marzo 2014. Tra i Gruppi approvati sono da citare per i temi trattati nella rivista:

- WaterCoRe AG on regional governance of water scarcity and drought issues (AG042);

- $\quad$ ESE - Ecosystem Services for Europe (AG052);

- RTWQM - Real Time Water Quality Monitoring (AG100);

- WaterReg - Water services regulation and governance in Europe (AG102);

- Mitigation of Water Stress in Coastal Zones by Sustainable Water Management (AG111);

- WIRE - Water \& Irrigated agriculture Resilient Europe (AG112);

- AugMent - Water Monitoring for Decision Support (AG124);

- MAR Solutions to Mar-ket -Managed Aquifer Recharge Strategies and Actions (AG128);

- DIS-SMEs - Demand-Driven Innovation Support for SMEs through the Network of National Water Partnerships (AG131);

- RESEWAM-O - Remote sensing for water management optimization (AG132).

L'intera iniziativa è ampiamente descritta alle pagine web: http://www.eip-water.eu/

\section{BIBLIOGRAFIA}

European Innovation Partnership,2013. http://ec.europa.eu/research/ innovation-union/index_en.cfm?pg=eip [ultimo accesso 30 Marzo 2014]

European Innovation Partnership on Water, 2013. http://www.eipwater.eu/ [ultimo accesso 30 Marzo 2014]

Innovation Union, 2013. http://ec.europa.eu/research/innovationunion/index_en.cfm [ultimo accesso 30 Marzo 2014] 